\title{
O Potencial fisiológico de sementes de cártamo tratados por termo terapia via calor úmido
}

\author{
Janine Farias Menegaes \\ Universidade Federal de Santa Maria (UFSM) \\ (janine_rs@hotmail.com) \\ Rogério Antonio Bellé \\ Universidade Federal de Santa Maria (UFSM) \\ (rogeriobelle@gmail.com) \\ Ubirajara Russi Nunes \\ Universidade Federal de Santa Maria (UFSM) \\ (russinunes@yahoo.com.br)
}

\begin{abstract}
Resumo: A cultura do cártamo (Carthamus tinctorius L.) apresenta interesse econômico com ampla aptidão de uso, desde alimentar a ornamental, oferecendo alta variabilidade de matérias-primas, com boa adaptabilidade de cultivo no Brasil. O objetivo deste trabalho foi avaliar o potencial fisiológico das sementes de cártamo tratadas por termoterapia via calor úmido. Visando o tratamento de semente como alternativa de agricultura com baixo impacto ambiental. $O$ delineamento utilizado foi inteiramente casualizado, em fatorial $4 \times 4$ (níveis de temperaturas e períodos de tempo), com oito repetições, sendo cada unidade experimental composta por 50 sementes. Os níveis de temperaturas foram compostos por $25,35,45$ e $55^{\circ} \mathrm{C}$ e os períodos de tempo de $5,10,15$ e 30 min. Avaliou-se o potencial fisiológico das sementes pelos testes de germinação, entropia, frequência relativa de germinação e teste de emergência. Observou-se que os tratamentos de sementes por termoterapia afetaram positivamente o potencial fisiológico das sementes de cártamo, incrementando o percentual de germinação e de emergência de plântulas. Conclui-se que a termoterapia é uma alternativa viável de tratamento de sementes para a cultura do cártamo, desde que utilize os níveis de temperaturas entre 25 a $45^{\circ} \mathrm{C}$, pelos períodos de tempo entre 5 e 15 minutos.
\end{abstract}

Palavras-chave: Carthamus tinctorius L; Frequências relativas; Germinação.

\section{Physiological potential of saffled seeds treated by thermotherapy through humid heat}

Abstract: The cultivation of safflower (Carthamus tinctorius L.) has an economic interest with a wide aptitude of use, from feeding to ornamental, offering high variability of raw materials, with good adaptability of cultivation in Brazil. The objective of this work was to evaluate the physiological potential of safflower seeds treated by thermotherapy via moist heat. Aiming at seed treatment as an agriculture alternative with low environmental impact. The design used was completely randomized, in a factorial $4 \times 4$ (temperature levels and time periods), with eight replications, each experimental unit consisting of 50 seeds. The temperature levels were composed by $25,35,45$ and $55^{\circ} \mathrm{C}$ and the time periods of $5,10,15$ and $30 \mathrm{~min}$. The physiological potential of the seeds was evaluated by germination, entropy, relative germination and emergency tests. It was observed that seed treatments 
by thermotherapy positively affected the physiological potential of safflower seeds, increasing the percentage of germination and seedling emergence. We conclude that thermotherapy is a viable alternative for treating seeds for the safflower culture, as long as it uses the temperature levels between 25 to $45^{\circ} \mathrm{C}$, for periods of time between 5 and 15 minutes.

Keywords: Carthamus tinctorius L; Relative frequencies; Germination.

\section{INTRODUÇÃO}

A cultura do cártamo (Carthamus tinctorius L.) apresenta interesse econômico em virtude da sua ampla aptidão de uso como matérias-primas desde alimentar a biodiesel, além do uso como planta ornamental (EMONGOR; OAGILE, 2017; MENEGAES et al., 2019a). Entre os diversos usos, destaca-se a extração de óleo das sementes, em média de $35 \%$ e rico em vitaminas C, E e betacaroteno, sendo utilizado, especialmente nas indústrias alimentícia, farmacêutica e petroleira (FAOSTAT, 2017; SANTOS; SILVA, 2015).

Cultivado em mais de 60 países como planta anual e oleífera, a cultura do cártamo apresenta alta rusticidade, desenvolvendo-se bem em ambientes com precipitações acima de $400 \mathrm{~mm}$ e amplitude térmica entre -7 a $40^{\circ} \mathrm{C}$, sendo responsivo a soma térmica que influência, diretamente na duração do ciclo de cultivo (CORONADO, 2010; EMONGOR; OAGILE; 2017). No Brasil, essa cultura ainda é pouco explorada comercialmente, mas pesquisas vêm demonstrando boa adaptabilidade de cultivo as condições edafoclimáticas dos Estados do Paraná (SAMPAIO et al., 2017) e do Rio Grande do Sul (BELLÉ et al., 2012; MENEGAES et al., 2019b) tanto para produção de sementes como flores de corte, respectivamente.

No estudo de manejo agronômico de uma cultura, a qualidade fitossanitária das sementes é de suma importância, em que muitas vezes necessita de tratamento, o qual contribui positivamente para a formação do estande de plantas no campo. Entre os métodos de tratamento de sementes, a termoterapia utiliza o binômio temperatura-tempo por imersão em água aquecida, devendo ser ajustada para cada espécie, de maneira que mantenha sua qualidade fisiológica e controle fitossanitário (PEREIRA et al., 2015). Esse método torna-se uma alternativa viável e com baixo impacto ambiental de tratamento de sementes, por não usar poluentes e nem emitir resíduos (LAZAROTTO et al., 2013; SCHNEIDER et al., 2015). 
A termoterapia tem se mostrado eficiente para a expressão do potencial fisiológico das sementes de diversas espécies oleíferas, com variados hábitos e importâncias, tais como, cumaru (Amburana cearensis A.C. Smith) (OLIVEIRA et al., 2011), mamona (Ricinus communis L.) (MARRONI et al., 2009), milho (Zea mays L.) (COUTINHO et al., 2007), pinhão-manso (Jatropha curcas L.) (SCHNEIDER et al., 2015), soja (Glycine max L.) (SANTOS et al., 2016), entre outros, oferecendo uma alternativa ao uso de produtos químicos, comumente, utilizados nos tratamentos das mesmas.

Neste contexto, em busca de uma alternativa para a agricultura de baixo impacto ambiental, o objetivo deste trabalho foi avaliar o potencial fisiológico das sementes de cártamo tratadas por termoterapia via calor úmido.

\section{MATERIAL E MÉTODOS}

O experimento foi conduzido no Laboratório Didático e de Pesquisas em Sementes do Departamento de Fitotecnia da Universidade Federal de Santa Maria

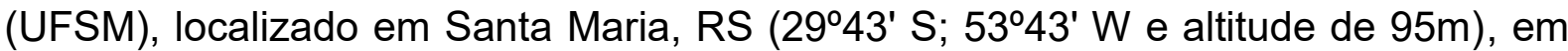
2018. O clima na região é subtropical úmido (Cfa), segundo a classificação de Köppen-Geiger.

As sementes de cártamo utilizados foram da cultivar Yellow Saffron, colhidas na safra 2017/2018, e armazenados em câmara fria ( $15^{\circ} \mathrm{C}$ e $40 \%$ UR) em sacos de papel kraft (tipo pardo de 1,0 kg), com grau de umidade médio de 9,0\% até a execução deste experimento.

O delineamento utilizado foi inteiramente casualizado, em esquema fatorial 4x4 (níveis de temperaturas e períodos de tempo), com oito repetições, sendo cada unidade experimental composta por 50 sementes. Os níveis de temperaturas foram compostos por $25,35,45$ e $55^{\circ} \mathrm{C}$ e os períodos de tempo de 5, 10, 15 e $30 \mathrm{~min}$. As sementes foram acondicionados em Becker de vidro de $500 \mathrm{~mL}$ contendo água destilada e este mantido em aparelho de banho-maria termodigital com água aquecida nas temperaturas e tempos conforme o fatorial supracitado. Previamente, as sementes foram embebidas em água destilada não aquecida, durante uma hora, para eliminação de bolsões de ar entre os tecidos mortos superficiais, facilitando a 
condução de calor nos tecidos das sementes (COUTINHO et al., 2007). Após o tratamento termoterápico as sementes foram postas para secar sobre papel-toalha a temperatura ambiente pelo período de $24 \mathrm{~h}$.

A caracterização do lote antes do tratamento e as avaliações qualitativas das sementes depois de tratadas foram pelos seguintes testes:

Teste padrão de germinação: com semeadura de oito repetições de 50 sementes, em rolo de papel de germinação, umedecido com água destilada na proporção de 2,5 vezes a massa do papel seco. Os rolos foram mantidos em germinador tipo Box Organism Development (BOD), com fotoperíodo de $24 \mathrm{~h}$ e temperatura de $25 \pm 2^{\circ} \mathrm{C}$. As avaliações foram realizadas aos 4 DAS (dias após a semeadura) para primeira contagem da germinação (PCG) e aos 14 DAS para germinação de plântulas normais (GER) (BRASIL, 2009). Para o índice de velocidade de germinação (IVG) (MAGUIRE, 1962) e para o tempo médio de germinação (TMG; dias) (FURBECK et al., 1993) foram realizadas avaliações diárias germinação. Utilizou-se como critério a germinação de plântulas normais as que apresentaram alongamento da raiz primária e emergência dos cotilédones (ABUD et al., 2010).

A frequência relativa de germinação (Fr) foi determinada pela metodologia de Labouriau e Valadares (1976), expressa na Equação 1.

$$
\mathrm{Fr}=\mathrm{ni} / \sum_{\mathrm{i}=1}^{\mathrm{k}} \mathrm{ni}
$$

em que: $\mathrm{Fr}$ = frequência relativa de germinação; ni = número de sementes germinadas por dia; $\Sigma \mathrm{ni}=$ número total de sementes germinadas. A entropia (índice de sincronização de germinação) foi determinado pela metodologia adaptada de Labouriau e Valadares (1976), expressa na Equação 2.

$$
E=\sum_{i=1}^{k} \text { fi. } \log 2 . f r
$$

em que: E: entropia informacional (bits); fr: frequência relativa de germinação; log2 logaritmo na base 2. 
No teste de emergência no campo: foram oito repetições de 50 sementes foram semeadas em linhas de $1 \mathrm{~m}$, com espaçamento entre si de 0,2 $\mathrm{m}$ e em sulcos de $0,03 \mathrm{~m}$ de profundidade.

Para o índice de velocidade de emergência (IVE) (MAGUIRE, 1962) e para o tempo médio de emergência (TME; dias) (FURBECK et al., 1993) foram realizadas avaliações diárias, e a avaliação da emergência foi realizada aos 14 DAS, com resultados expressos em percentagem de emergência. Utilizou-se como critério de emergência de plântulas as com desenvolvimento completo dos cotilédones e epicótilo (ABUD et al., 2010).

Para as variáveis de germinação e emergência das plântulas no campo, utilizou-se como referência a Instrução Normativa $n^{\circ} .45 / 2013$ do Ministério da Agricultura, Pecuária e Abastecimento (MAPA) para a cultura do girassol (Helianthus annuus L.), por pertencer à mesma família botânica do cártamo (Asteraceae), sendo exigidos valores $65 \%$ a $70 \%$ (BRASIL, 2013).

Os dados expressos em percentagem foram transformados em arcoseno $\sqrt{x / 100}$. Análises de variância (ANOVA) dos dados e a comparação de médias pelo teste de Scott-Knott $(p<0,05)$, foram realizadas com o auxílio do programa SISVAR (FERREIRA, 2014).

\section{RESULTADOS E DISCUSSÃO}

Verificou-se que o potencial fisiológico do lote inicial de sementes de cártamo utilizado, neste trabalho, foi em média de $66 \%$ de germinação e $76 \%$ de emergência no campo (Tabela 1), caracterizando-se como possível lote comercial segundo os padrões do MAPA (BRASIL, 2013).

Tabela 1 - Caracterização inicial do lote de sementes de cártamo (Carthamus tinctorius L.).

\begin{tabular}{cc}
\hline Caracterização inicial & Parâmetros \\
\hline Primeira contagem da germinação (PCG) & $39 \%$ \\
Germinação (GER) & $66 \%$ \\
Índice de velocidade de germinação (IVG) & 93,166 \\
Tempo médio de germinação (TMG) & 8,1 dias
\end{tabular}


Entropia

Emergência no campo (ECP)

Índice de velocidade de emergência (IVE)

Tempo médio de emergência (TME)
1,22 bits

$76 \%$

56,420

12,1 dias

A primeira contagem da germinação (PCG) verificada aos 4 DAS para o lote inicial foi de 39\% (Tabela 1), esse parâmetro é um indicativo de vigor das sementes para um estabelecimento rápido da lavoura no campo. Na Tabela 2, verificou-se proximidade do percentual de PCG para as sementes submetidas aos tratamentos de termoterapia via calor úmido, entre os níveis de temperaturas de 25 a $45^{\circ} \mathrm{C}$ pelos períodos de tempo de 5 a15 minutos.

Tabela 2 - Primeira contagem da germinação (PCG), germinação (GER), índice de velocidade de germinação (IVG), tempo médio de germinação (TMG), entropia, emergência no campo (ECP), índice de velocidade de emergência (IVE) e tempo médio de emergência

(TME) de sementes de cártamo (Carthamus tinctorius L.) submetidos à termoterapia via calor úmido.

\begin{tabular}{|c|c|c|c|c|c|c|c|c|c|c|}
\hline \multirow{2}{*}{$\begin{array}{l}\text { Tempo } \\
\text { (min.) }\end{array}$} & \multicolumn{5}{|c|}{ Temperatura $\left({ }^{\circ} \mathrm{C}\right)$} & \multicolumn{5}{|c|}{ Temperatura $\left({ }^{\circ} \mathrm{C}\right)$} \\
\hline & 25 & 35 & 45 & 55 & Média & 25 & 35 & 45 & 55 & Média \\
\hline & \multicolumn{5}{|c|}{ PCG (\%) } & \multicolumn{5}{|c|}{ GER (\%) } \\
\hline 5 & $41^{*} \mathrm{Aa}$ & $40 \mathrm{Ab}$ & $41 \mathrm{Aa}$ & $30 \mathrm{Ba}$ & 38 & 70 * $\mathrm{Ba}$ & $77 \mathrm{Aa}$ & $73 \mathrm{Ba}$ & $50 \mathrm{Ca}$ & 67 \\
\hline 10 & $40 \mathrm{Aa}$ & $39 \mathrm{Ab}$ & $40 \mathrm{Aa}$ & $15 \mathrm{Bb}$ & 33 & $71 \mathrm{Aa}$ & $73 A b$ & $73 \mathrm{Aa}$ & $38 \mathrm{Bb}$ & 64 \\
\hline 15 & $40 \mathrm{Ba}$ & $44 \mathrm{Aa}$ & $40 \mathrm{Ba}$ & $10 \mathrm{Cb}$ & 33 & $72 \mathrm{Ba}$ & $71 \mathrm{Bb}$ & $75 \mathrm{Aa}$ & $32 \mathrm{Cc}$ & 62 \\
\hline 30 & $37 \mathrm{Ab}$ & $28 \mathrm{Bc}$ & $30 \mathrm{Bb}$ & $6 \mathrm{Cc}$ & 25 & $62 A b$ & $60 \mathrm{Bc}$ & $63 \mathrm{Ab}$ & $30 \mathrm{Cc}$ & 54 \\
\hline Média & 39 & 38 & 38 & 15 & & 69 & 70 & 71 & 38 & \\
\hline \multirow[t]{2}{*}{ CV (\%) } & \multicolumn{5}{|c|}{7,06} & \multicolumn{5}{|c|}{7,36} \\
\hline & \multicolumn{5}{|c|}{ IVG } & \multicolumn{5}{|c|}{ TMG (dias) } \\
\hline 5 & 78,842 * $\mathrm{Aa}$ & $78,456 \mathrm{Aa}$ & $72,765 \mathrm{Bb}$ & $38,322 \mathrm{Ca}$ & 67,096 & $8,7^{\mathrm{ns}}$ & 8,2 & 8,9 & 8,1 & $8,5 \mathrm{~b}$ \\
\hline 10 & $74,567 \mathrm{Ab}$ & $73,577 \mathrm{Ab}$ & $73,123 \mathrm{Ab}$ & $27,214 \mathrm{Bc}$ & 62,120 & 8,7 & 8,5 & 8,9 & 8,1 & $8,6 \mathrm{~b}$ \\
\hline 15 & $61,538 \mathrm{Bc}$ & $74,497 \mathrm{Ab}$ & $76,171 \mathrm{Aa}$ & $28,702 \mathrm{Cc}$ & 60,227 & 9,1 & 8,8 & 8,9 & 8,3 & $8,8 \mathrm{a}$ \\
\hline 30 & $76,988 \mathrm{Ab}$ & $70,540 \mathrm{Bc}$ & $59,124 \mathrm{Cc}$ & $30,390 \mathrm{Db}$ & 59,261 & 8,7 & 7,9 & 9,1 & 8,0 & $8,4 \mathrm{~b}$ \\
\hline Média & 72,984 & 74,267 & 70,296 & 31,157 & & $8,8 \mathrm{~A}$ & $8,3 \mathrm{~B}$ & $8,9 \mathrm{~A}$ & $8,1 \mathrm{~B}$ & \\
\hline \multirow[t]{2}{*}{ CV (\%) } & \multicolumn{5}{|c|}{5,86} & \multicolumn{5}{|c|}{4,47} \\
\hline & \multicolumn{5}{|c|}{ Entropia da germinação (bits) } & \multicolumn{5}{|c|}{ ECP (\%) } \\
\hline 5 & $1,04{ }^{*} \mathrm{Aa}$ & $1,00 \mathrm{Aa}$ & $0,99 \mathrm{Aa}$ & $0,19 \mathrm{Bb}$ & 0,80 & $76{ }^{*} \mathrm{Bb}$ & $77 \mathrm{Ba}$ & $80 \mathrm{Aa}$ & $62 \mathrm{Ca}$ & 74 \\
\hline 10 & $0,95 \mathrm{Aa}$ & $0,97 \mathrm{Aa}$ & $0,99 \mathrm{Aa}$ & $0,28 \mathrm{Ba}$ & 0,80 & $78 \mathrm{Ba}$ & $75 \mathrm{Ba}$ & $81 \mathrm{Aa}$ & $58 \mathrm{Ca}$ & 73 \\
\hline 15 & $0,80 \mathrm{Bb}$ & 0,99 Aa & $1,01 \mathrm{Aa}$ & $0,29 \mathrm{Ca}$ & 0,77 & $79 \mathrm{Ba}$ & $72 \mathrm{Cb}$ & $83 \mathrm{Aa}$ & $46 \mathrm{Db}$ & 70 \\
\hline 30 & $1,03 \mathrm{Aa}$ & $0,83 \mathrm{Bb}$ & $0,76 \mathrm{Bb}$ & $0,04 \mathrm{Cc}$ & 0,67 & $76 \mathrm{Ab}$ & $70 \mathrm{Bb}$ & $76 \mathrm{Ab}$ & $43 \mathrm{Cb}$ & 66 \\
\hline Média & 0,96 & 0,95 & 0,94 & 0,20 & & 77 & 74 & 80 & 52 & \\
\hline \multirow[t]{2}{*}{ CV (\%) } & \multicolumn{5}{|c|}{8,54} & \multicolumn{5}{|c|}{6,61} \\
\hline & \multicolumn{5}{|c|}{ IVE } & \multicolumn{5}{|c|}{ TME (dias) } \\
\hline 5 & $56,122{ }^{*} \mathrm{Aa}$ & $53,470 \mathrm{Ba}$ & $55,351 \mathrm{Aa}$ & $38,955 \mathrm{Ca}$ & 50,975 & $12,5^{\mathrm{ns}}$ & 12,6 & 12,6 & 13,0 & 12,7 \\
\hline
\end{tabular}


O Potencial fisiológico de sementes de cártamo tratados por termo terapia via calor úmido

\begin{tabular}{ccccccccccc}
10 & $56,856 \mathrm{Aa}$ & $52,506 \mathrm{Ba}$ & $54,035 \mathrm{Ab}$ & $37,006 \mathrm{Ca}$ & 50,101 & 12,3 & 12,9 & 13,2 & 13,2 & $12,9 \mathrm{a}$ \\
15 & $54,293 \mathrm{Bb}$ & $53,373 \mathrm{Ba}$ & $56,597 \mathrm{Aa}$ & $34,272 \mathrm{Cb}$ & 49,634 & 12,9 & 12,5 & 12,6 & 12,8 & $12,7 \mathrm{a}$ \\
30 & $54,424 \mathrm{Ab}$ & $52,187 \mathrm{Aa}$ & $53,108 \mathrm{Ab}$ & $32,946 \mathrm{Bc}$ & 48,166 & 12,5 & 12,6 & 13,0 & 13,0 & $12,8 \mathrm{a}$ \\
Média & 55,424 & 52,884 & 54,773 & 35,795 & & $12,5 \mathrm{~A}$ & $12,6 \mathrm{~A}$ & $12,8 \mathrm{~A}$ & $13,0 \mathrm{~A}$ & \\
\hline CV (\%) & & & 5,47 & & & & & 6,02 & &
\end{tabular}

* interação significativa $\mathrm{e}^{\text {ns }}$ interação não significativa dos fatores. Teste de médias não seguidas pela mesma letra, maiúsculas na linha e minúsculas na coluna, diferem pelo teste Scott-Knott $(p<0,05)$. CV: Coeficiente de variação.

Observou-se que o percentual de germinação (GER) e de emergência no campo (ECP) das sementes de cártamo submetidas aos tratamentos de termoterapia foram acima de $70 \%$, para os níveis de temperaturas entre 25 a $45^{\circ} \mathrm{C}$, para a GER pelos períodos de tempo entre 5 e 15 minutos e para a ECP pelos períodos de tempo de 5 a 30 minutos (Tabela 2). Ambos os parâmetros de GER e ECP, confirmam que os tratamentos por termoterapia mantiveram o lote de sementes de acordo com os padrões preconizados pelo MAPA (BRASIL, 2013), além de promoveram um incremento tanto no percentual de germinação como de emergência de plântulas após o tratamento das sementes.

Pereira et al. (2015) e Araújo et al. (2018) apontam que a eficiência da termoterapia como tratamento de sementes ocorre uma vez que a mesma, fundamenta-se no ajuste ideal do binômio temperatura-tempo, para a preservação do potencial fisiológico das sementes tratadas. Por isso Coutinho et al. (2007), indicam que há necessidade de embeber as sementes em água ambiente (não aquecida) com o intuito de eliminar de bolsões de ar entre os tecidos mortos superficiais, facilitando a condução do calor e evitando danos maiores as membranas.

Os índices de velocidade de germinação (IVG) e de emergência (IVE), do lote inicial das sementes de cártamo foram de 93,166 e 56,420 (Tabela 1), respectivamente. Todavia, observou-se que ambos os índices foram menores para as sementes submetidas aos tratamentos de termoterapia (Tabela 2). Para Borghetti e Ferreira (2004), os índices IVG e IVE, representam a velocidade que as sementes levam para reorganizar as membranas. Na presente pesquisa, observou-se que a termoterapia afetou negativamente essa velocidade de reorganização das membranas, sobretudo para o nível de temperatura a $55^{\circ} \mathrm{C}$, em todos os períodos de exposição ao calor úmido. 
Os tempos médios de germinação (TMG) e de emergência (TME), foram próximos de 8 e 12 DAS, respectivamente, tanto para o lote inicial de sementes de cártamo como para as submetidas aos tratamentos de termoterapia. Marcos-Filho (2015) relata que a manutenção da integridade do sistema de membranas é fundamental para a preservação da vitalidade e da viabilidade das sementes, especialmente as submetidas ao estresse por calor, levando maior tempo para reorganização deste sistema. Menegaes et al. (2019a) verificaram que quando as sementes de cártamo são expostas a temperatura acima de $45^{\circ} \mathrm{C}$ por mais de 30 minutos por calor úmido, mais lento é o desencadeamento dos processos metabólicos das dessas sementes, afetando negativamente a velocidade e o tempo de germinação e de emergência de plântulas.

Figura 1 - Frequências relativas ( $\mathrm{Fr}$; \%) de sementes germinadas de cártamo (Carthamus tinctorius $\mathrm{L}$.) do lote inicial e as submetidas a termoterapia via calor úmido. NTG: número total de sementes germinadas (unidades), TMG: tempo médio de germinação (dias). 
a) Caracterização inicial

Caracterização inicial TMG
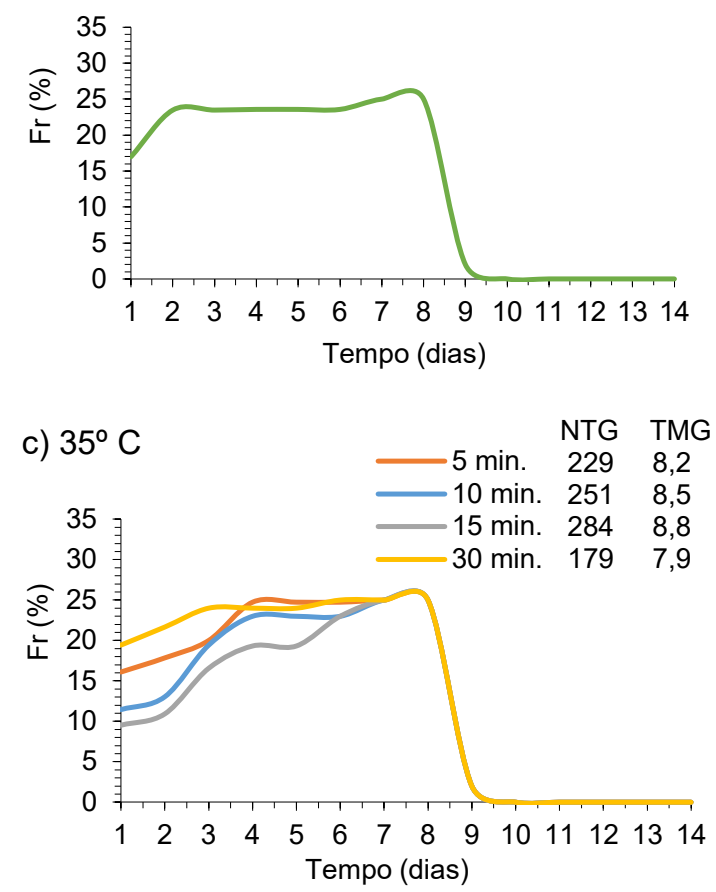

e) $55^{\circ} \mathrm{C}$

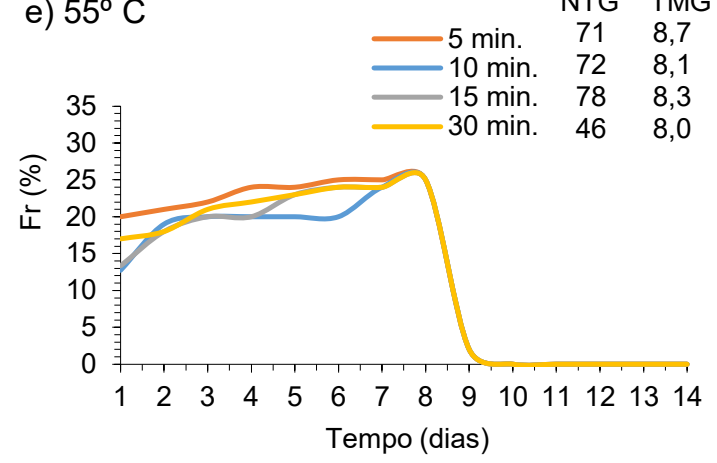

b) $25^{\circ} \mathrm{C}$

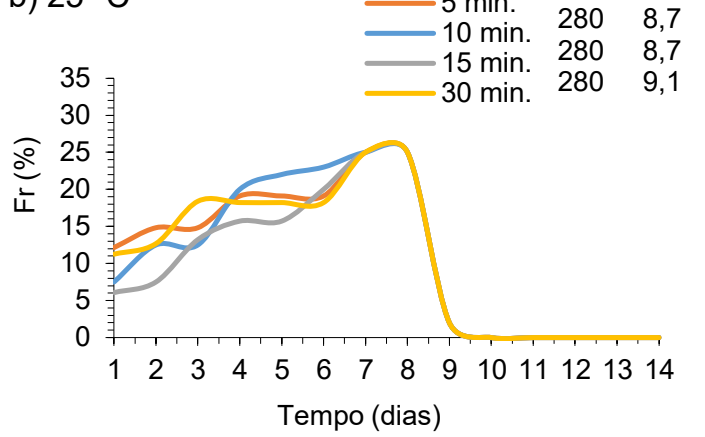

d) $45^{\circ} \mathrm{C}$

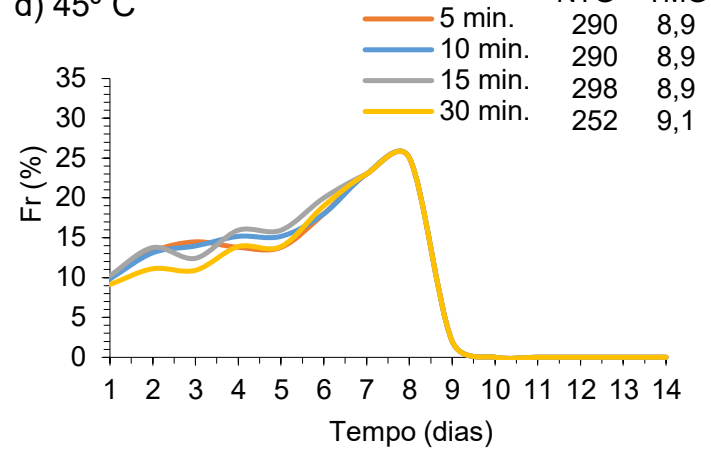

A sincronização da germinação verificada pela entropia, indica que os níveis de temperatura entre 25 a $45^{\circ} \mathrm{C}$ não são prejudiciais a germinabilidade das sementes de cártamo, pois a esses níveis os valores de entropia fica próximo ao do lote inicial com valor de 1,22 bits em temperatura ambiente (Tabelas 1 e 2). Já para todos os períodos de exposição a temperatura de $55^{\circ} \mathrm{C}$, observou-se que os valores foram muito reduzidos, indicando o processo de deterioração destas sementes. Segundo Lopes e Franke (2011), os valores de entropia é confirmado pela forma da distribuições das frequências relativas da germinação, onde os níveis de temperatura representam sinais de ambientação ao estresse térmico. 
Figura 2 - Frequências relativas (Fr; \%) de plântulas emergidas de cártamo (Carthamus tinctorius L.) do lote inicial e as submetidas a termoterapia via calor úmido. NTE: número total de plântulas emergidas (unidades), TME: tempo médio de emergência (dias).

a) Caracterização inicial
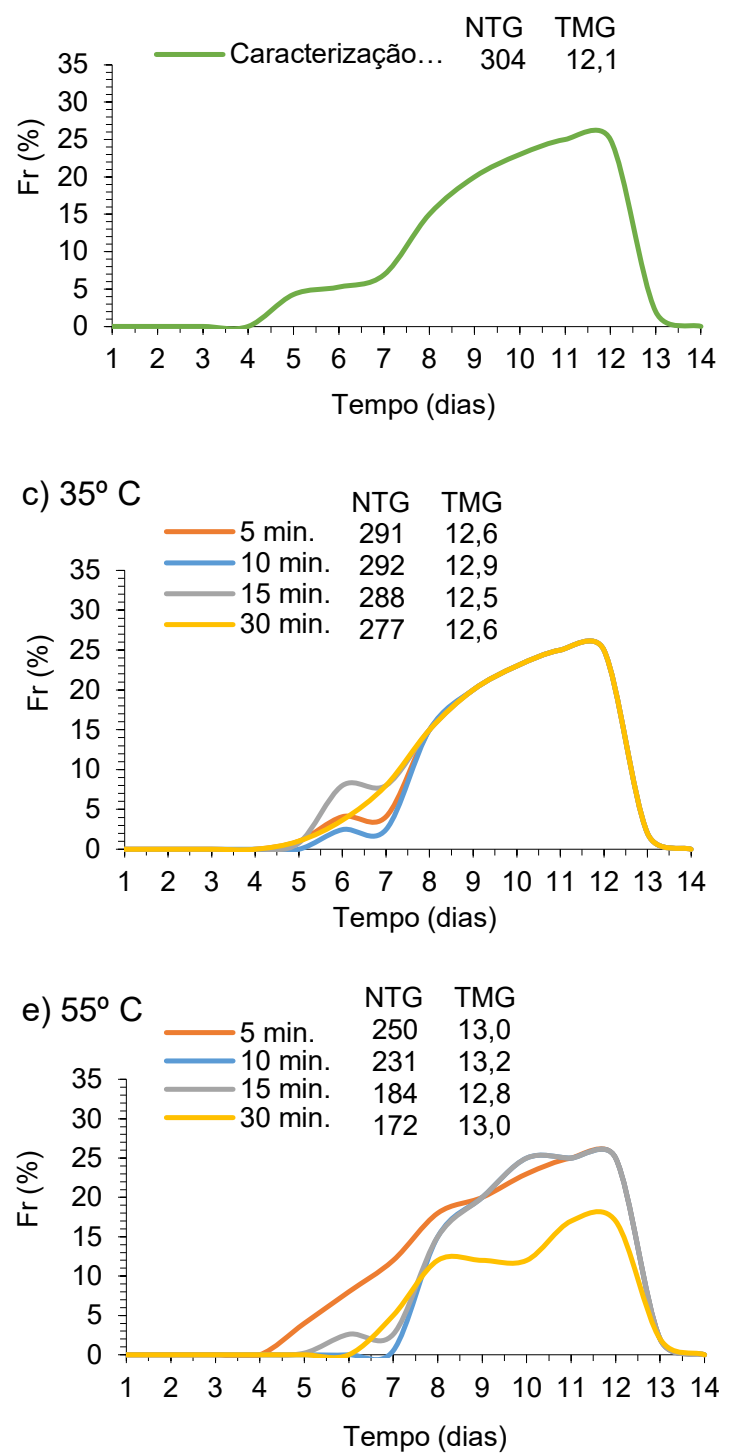
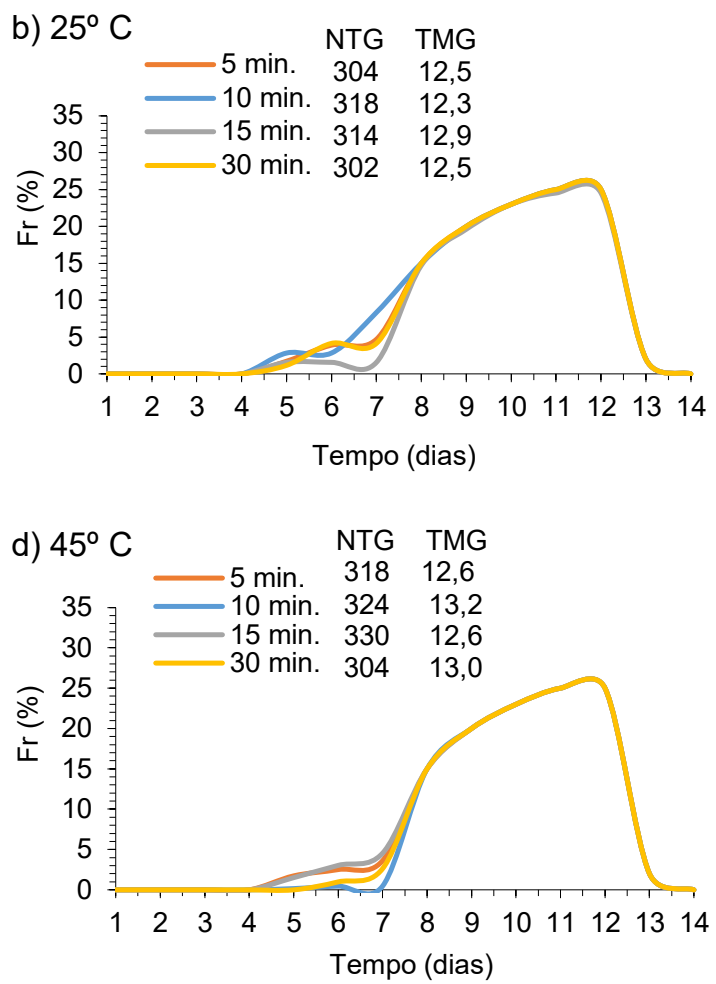

Nas Figuras 1 e 2, as frequências relativas de sementes germinadas e de emergência de plântulas de cártamo se distribuíram similarmente obtendo os picos de sementes germinadas e de emergência de plântulas próximo aos 8 e 12 DAS, respectivamente. As coincidências dos picos de germinação e de emergências com proximidade dos TMG e TME, tanto para as sementes do lote inicial (Tabela 1) como para as sementes que receberam o tratamento de termoterapia (Tabela 2), demonstram uma homogeneidade do potencial fisiológico dessas sementes.

Menegaes et al. (2019a) verificaram que as sementes de cártamo são tolerantes a tratamentos por calor até $45^{\circ} \mathrm{C}$, sem prejuízo ao potencial fisiológico. 
Lopes e Franke (2011) observaram que a distribuição das frequências da germinação de sementes cornichão (Lotus corniculatus L.) seguiu uma moda, com implicação direta do TMG para uma germinação uniforme.

Silva et al. (2017) verificaram que as diferentes condições térmicas não afetaram as frequências relativas de germinação das sementes de tamarindo (Tamarindus indica L.), sendo as sincronicidade dos picos foram atribuídos ao potencial fisiológico das mesmas. Menegaes et al. (2018; 2019c) atribuíram as coincidências dos picos de germinação de duas espécies de celosia (Celosia argentea L. e C. cristata L.) tanto as submetidas a diferentes condições de temperatura e luz, quando as submetidas a diferentes períodos de armazenamento, ao potencial fisiológico das sementes.

\section{CONCLUSÃO}

O tratamento de sementes por termoterapia via calor úmido torna-se uma alternativa viável e de baixo impacto ambiental para a cultura de cártamo, incrementando ainda o seu potencial fisiológico tanto para a germinação como para emergência no campo. Dentre os tratamentos, recomenda-se realizar a termoterapia entre as temperaturas de 25 a $45^{\circ} \mathrm{C}$, pelos períodos de tempo entre 5 e 15 minutos.

\section{AGRADECIMENTOS}

A CAPES (Coordenação de Aperfeiçoamento de Pessoal de Nível Superior) pelo incentivo e financiamento deste trabalho e, ao Programa de Pós-Graduação em Agronomia da Universidade Federal de Santa Maria. Ao CNPq pela bolsa de produtividade ao professor orientador deste trabalho.

\section{REFERÊNCIAS}

ABUD, H. F.; GONÇALVES, N. G.; REIS, R. G. E. S.; GALLÃO, M. I.; INNECCO, R. Morfologia de sementes e plântulas de cártamos. Revista Ciência Agronômica, Fortaleza, v. 41, n. 2, p. 259-265, 2010. 
ARAÚJO, F. S.; SOUSA, N. A; ALVES, E. C.; FARIAS, O. R.; NASCIMENTO, L. C.; BRUNO, R. L. A.; PACHECO, M. V. Tratamento térmico úmido em sementes de Acacia mangium. Revista de Ciências Agrárias, Lisboa, v. 41, n. 3, p. 702-708, 2018.

BELLÉ, R. A.; ROCHA, E. K.; BACKES, F. A. A. L.; SCHWAB, N. T. Safflower grown in different sowing dates and plant densities. Ciência Rural, Santa Maria, v. 42, n. 12, p. 2145-2152. 2012.

BORGHETTI, F.; FERREIRA, A. G. Interpretação de resultados de germinação. In: FERREIRA, A. G.; BORGHETTI, F. (Org.). Germinação: do básico ao aplicado. Porto Alegre: Artmed, 2004. p. 209-222.

BRASIL. Ministério da Agricultura, Pecuária e Abastecimento. Instrução Normativa n. 45. Brasília. MAPA. 2013, 38p.

BRASIL. Ministério da Agricultura, Pecuária e Abastecimento. Regras para Análise de Sementes. Brasília: MAPA, 2009. 395p.

CORONADO, L. M. El cultivo del cártamo (Carthamus tinctorius L.) en México. Ciudade Obregon: SGI. 2010, 96p.

COUTINHO, W. M.; SILVA-MANN, R.; VIERIA, M. G. G. C.; MACHADO, C. F.; MACHADO, J. C. Qualidade sanitária e fisiológica de sementes de milho submetidas à termoterapia e condicionamento fisiológico. Fitopatologia Brasileira, Brasília, v. 32 , n. 6, p. 458-4654, 2007.

EMONGOR, V.; OAGILE, O. Safflower production. Botswana: The Regional Universities Forum for Capacity Building in Agriculture - RUFORUM. 2017. 67p.

FAOSTAT. FOOD AND AGRICULTURE ORGANIZATION OF THE UNITED NATIONS STATISTICS DIVISION. Crops: Safflower. 2017. Disponível em: <http://faostat3.fao.org/browse/Q/QC/E>. Acesso em: 02 mar. 2019.

FERREIRA, D. F. Sisvar: A guide for its bootstrap procedures in multiple comparisons. Ciência e Agrotecnologia, v.38, n. 2, p.109-112, 2014.

FURBECK, S. M.; BOURLAND, F. M.; WATSON, C. E. Relationship of seed and germination measurements with resistance to seed weathering cotton. Seed Science and Technology, v. 21, n. 3, p. 505-512, 1993.

LABOURIAU, L. G.; VALADARES, M. E. B. On the germination of seeds Calotropis procera (Ait.) Ait.f. Anais da Academia Brasileira de Ciências, Rio de Janeiro, v. 48, n. 2, p. 263-284, 1976.

LAZAROTTO, M.; MEZZOMO, R.; MACIEL, C.G.; BOVOLINI, M. P.; MUNIZ, M. F. B. Tratamento de sementes de canafístula via calor úmido. Revista Ciência Agraria, Manaus, v.56, n.3, p.268-273, 2013. 
LOPES, R. R.; FRANKE, L. B. Aspectos térmico-biológicos da germinação de sementes de cornichão anual sob diferentes temperaturas. Revista Brasileira de Zootecnia, v. 40, n. 10, p.2 091-2096, 2011

MAGUIRE, J. D. Speed of germination aid in selection and evaluation for seedling emergence and vigor. Crop Science, v. 2, n. 2, p.176-177, 1962.

MARCOS-FILHO, J. Fisiologia de sementes de plantas cultivadas. ABRATES: Londrina, 2015. 650p.

MARRONI, I. V.; ZANATTA, Z. G. C. N.; JUNIRO, J. G. C.; UENO, B.; MOURA, A. B. Efeito do tratamento com calor seco e água quente sobre a germinação e controle de micro-organismos associados às sementes de mamoneira. Arquivos do Instituto Biológico, São Paulo, v.76, n.4, p.761-767, 2009.

MENEGAES, J. F.; BARBIERI, G. F.; BELLÉ, R. A.; NUNES, U. R. Photoblastic and temperatures in the germination of cockscomb seeds. Ornamental Horticulture, Campinas, v. 24, n. 4, p. 408-414, 2018.

MENEGAES, J. F.; BARBIERI, G. F.; BELLÉ, R. A.; NUNES, U. R. Physiological and sanitary quality of cockscomb seeds stored for different periods. Ornamental Horticulture, Campinas, v. 25, n. 1, p. 34-41, 2019c.

MENEGAES, J. F.; LIDÓRIO, H. F.; BELLÉ, R. A.; LOPES, S. J.; BACKES, F. A. A. L.; NUNES, U. R. Post-harvest of safflower flower stems harvested at different times and submitted to different preservative solutions. Ornamental Horticulture, Campinas, v. 25, n. 1, p. 87-96, 2019b.

MENEGAES, J. F.; NUNES, U. R.; BELLÉ, R. A.; LOPES, S. J.; FERNANDES, T. S.; LUDWIG, E. J.; ZINI, P. B.; BARBIERI, G. F. Thermotherapy via humid heat for the treatment of safflower seeds. Journal of Agricultural Science, Ontario, v. 11, n. 11, p.30-40, 2019a.

OLIVEIRA, M. D. M.; NASCIMENTO, L. C.; ALVES, E. U.; GONÇALVES, E. P.; GUEDES, R. S.; NETO, J. J. S. Qualidade sanitária e fisiológica de sementes de Amburana cearenses A.C. Smith submetidas à termoterapia e tratamento químico. Acta Scientiarum Agronomy, Maringá, v. 33, n. 1, p. 45-50, 2011.

PEREIRA, R. B.; SILVA, P. P.; NASCIMENTO, W. M.; PINHEIRO, J. B. Tratamento de sementes de hortaliças. Circular Técnica 140. Brasília: EMBRAPA. 2015. 16p.

SAMPAIO, M. C.; SANTOS, R. F.; BASSEGIO, D.; VASCONSELOS, E. S.; SILVEIRA, L.; LENZ, N. B. G.; LEWADOSKI, C. F.; TOKURO, L. K. Effect of plant density on oil yield of safflower. African Journal of Agricultural Research, v. 12, n. 25, p. 2147-2152, 2017.

SANTOS, L. A.; FARIA, D. R.; CACILDA, M.; MAREK, J.; DUHATSCHEK, E.; MARTNICHEN, D. Radioterapia e termoterapia como tratamentos de sementes de soja. Brazilian Journal of Applied Technology for Agricultural Science, Guarapuava, v. 9, n .2, p. 37-44, 2016. 
SANTOS, R. F.; SILVA, M. A. Carthamus tinctorius L.: Uma alternativa de cultivo para o Brasil. Acta Iguazu, Cascavel, v.4, n.1, p. 26-35, 2015.

SCHNEIDER, C. F.; GUSATTO, F. G.; MALAVASI, M. M.; STANGARLIN, J. R.; MALAVASI, U. C. Termoterapia na qualidade fisiológica e sanitária de sementes armazenadas de pinhão-manso. Semina: Ciências Agrárias, Londrina, v. 36, n. 1, p. 47-56, 2015.

SILVA, D. D. A; MACHADOM C. G.; CRUZ, S. C. S.; VESPUCCI, I. L.; ARAUJO, Y. J. D. Temperatura e substrato para o teste de germinação de sementes de tamarindo. Espacios, Cararacas, v. 38, n. 14, p. 4-15, 2017. 